\title{
Studies of the plume emission during the femtosecond and nanosecond ablation of graphite in nitrogen
}

\author{
Gareth M. Fuge and Michael N. R. Ashfold ${ }^{a)}$ \\ School of Chemistry, University of Bristol, Bristol BS8 1TS, United Kingdom \\ Simon J. Henley \\ Advanced Technology Institute, School of Electronics and Physical Sciences, University of Surrey, Guildford \\ GU2 7XH, United Kingdom
}

(Received 25 March 2005; accepted 18 November 2005; published online 12 January 2006)

\begin{abstract}
Comparative studies of the pulsed laser ablation of graphite in 20 mTorr of $\mathrm{N}_{2}$ using both $15 \mathrm{~ns}$ and $450 \mathrm{fs}$ pulses at a wavelength of $248 \mathrm{~nm}$ are reported. Emissions from the resulting ablation plumes, and from collisions with ablated material and the background $\mathrm{N}_{2}$ gas molecules, have been investigated by wavelength-, space-, and time-resolved optical emission spectroscopy (OES), and the observations correlated with the results of the analyses of films formed when such material is incident on a silicon substrate. Wavelength-dispersed spectra of the plume arising in nanosecond ablation reveal $\mathrm{C}_{\mathrm{I}}, \mathrm{C}$ II, and $\mathrm{C}_{2}$ emissions-concentrated close to the target-and, at greater distances, strong $\mathrm{CN}$ and weak $\mathrm{N}_{2}^{+}$emissions. $\mathrm{N}_{2}^{+}(B-X)$ emission dominates in the case of femtosecond ablation. Time-gated imaging studies have allowed estimation of propagation velocities for these various emissions. Possible production routes for secondary emitters such as CN and $\mathrm{N}_{2}^{+}$are discussed, and arguments presented to show that measurements of the apparent propagation "velocities" of such emissions are unlikely to provide meaningful measures of the velocities (or energies) with which these carriers impact on a substrate surface. Laser Raman spectroscopy confirms nitrogen incorporation within the films grown by both nanosecond and femtosecond ablations; the former films are deduced to be both thicker and to have higher $\mathrm{N}$ content-findings that accord with the OES analyses. () 2006 American Institute of Physics. [DOI: $10.1063 / 1.2158500]$
\end{abstract}

\section{INTRODUCTION}

Nitrogen-containing, hydrogen-free, diamondlike carbon (DLC) - henceforth identified as $\mathrm{CN}_{x}$ - films have been investigated quite extensively over the past decade. Most such films have been produced by deposition on a suitable substrate following nanosecond ultraviolet (UV) pulsed laser ablation (PLA) of a graphite target in a low-pressure background of nitrogen gas. ${ }^{1-3}$ The resulting films have been suggested to offer a number of benefits in comparison with pure carbon films, including increased resilience to mechanical wear ${ }^{4}$ and reduced surface roughness. ${ }^{5}$ Such $\mathrm{CN}_{x}$ films have been subjected to many different analysis techniques in an attempt to understand the film characteristics and to correlate these with properties of the ablation plume from which the films are formed, e.g., the relative number densities and the velocity (and energy) distributions of the various species in the plume. ${ }^{6,7}$ Femtosecond (as compared with nanosecond) PLA offers somewhat different deposition conditionse.g., less material transfer per pulse and reduced laser-plume interactions, but the initial kinetic-energy distribution of the ejected material is higher, as is its average degree of ionization. ${ }^{8}$ Material and structural properties of nitrogenfree DLC thin films deposited by femtosecond PLA of graphite in vacuum have been investigated ${ }^{9-13}$ and deduced to have an $s p^{3}$ content of $\sim 70 \%$ and to contain some nanocrys-

\footnotetext{
${ }^{\text {a) }}$ Author to whom correspondence should be addressed; fax: (117)-9250612; electronic mail: mike.ashfold@bris.ac.uk
}

talline diamond phase within an amorphous matrix. ${ }^{10}$ The high $s p^{3}$ content has been attributed to the increased kinetic, and thus impact, energy of the ejected particles arising in PLA induced with shorter pulse durations. ${ }^{8}$ Possible advantages of producing $\mathrm{CN}_{x}$ thin films using subpicosecond laser pulses have been investigated by a few groups, ${ }^{14-17}$ not least with the aim of reducing the particulate density within and on the resulting film.

Here we report wavelength-, time-, and spatially resolved measurements of the optical emissions that accompany the plume arising in the $248 \mathrm{~nm}$ PLA of graphite in low background pressures of nitrogen, together with limited analyses (visible Raman spectroscopy and scanning electron microscopy) of the as-deposited films. Results obtained using both nanosecond and femtosecond laser pulses are compared and contrasted, and possible implications for $\mathrm{CN}_{x}$ film growth discussed.

\section{EXPERIMENT}

The ablation apparatus, shown in Fig. 1, consisted of a high-vacuum stainless-steel chamber which was evacuated by a turbomolecular pump, backed by a rotary pump, to a base pressure of $\sim 1 \times 10^{-6}$ Torr. The chamber was back filled with 20 mTorr of $\mathrm{N}_{2}$ during all ablation and deposition experiments reported here. The incident laser beam was directed through one of the chamber side arms (which was sealed with a quartz window) and focused $[30 \mathrm{~cm}$ focal length (fl) biconvex lens] onto the target at $45^{\circ}$ to the surface 


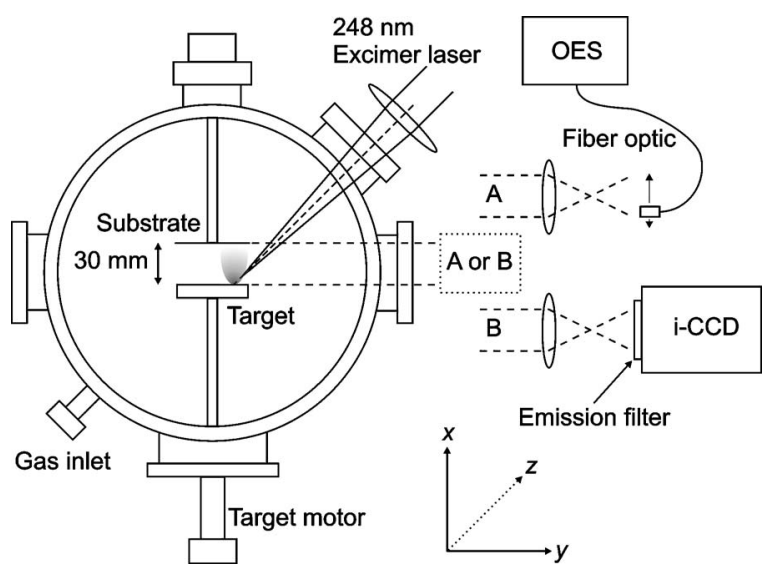

FIG. 1. Schematic illustration of the experimental apparatus. Alternative arrangements used for collecting (A) wavelength-resolved and (B) timegated OES are depicted to the right.

normal (here defined as the $x$ axis). The target, a 2 in. diameter disk of highly oriented pyrolytic graphite (HOPG, 99.9\% purity), was rotated to minimize repeated ablation of the same spot. Pulses of $248 \mathrm{~nm}$ radiation with respective full width at half maxima (FWHM) of 15 ns and 450 fs were generated using two different laser systems, as described previously. ${ }^{8,18}$ The respective pulse energies were $\sim 47 \mathrm{~mJ} / 15 \mathrm{~ns}$ pulse and $\sim 8.2 \mathrm{~mJ} / 450 \mathrm{fs}$ pulse. The two laser outputs have different spatial profiles, beam divergences, etc., the spot sizes on the target were thus very different. The incident fluence in each case was similar $\left(\sim 12.5 \mathrm{~J} \mathrm{~cm}^{-2}\right)$, though the respective power densities were of course very different: $\sim 6.3 \times 10^{8}$ and $\sim 2.8 \times 10^{13} \mathrm{~W} \mathrm{~cm}^{-2}$.

Optical emission accompanying the PLA process was viewed along the $y$ axis (i.e., parallel to the front face of the target), as a function of $x$ and in the $x z$ plane, as described previously. ${ }^{18}$ The variation of the optical emission spectrum with $x$ was studied using arrangement (A) in Fig. 1. The emission was focused onto the entrance of a translatable quartz single fiber, the exit of which was arranged to about the entrance slit of a $0.32 \mathrm{~m}$ spectrometer coupled to an intensified charge-coupled device (i-CCD). Evolving spatial distributions of selected emitting species were measured with arrangement (B). In such studies, the total emission, or just the fraction that is transmitted through an appropriate narrow bandpass interference filter, was focused (2:1 magnification) directly onto the $1024 \times 256$ diode array of the i-CCD (thus enabling use of its full response range which extends to $\lambda$ $>900 \mathrm{~nm}$ ). The initial delay before an i-CCD image could be collected was limited to $t \geqslant 120 \mathrm{~ns}$ by the response time of the available electronics.

Films were deposited on $n$-type single-crystal (100) silicon substrates, which were mounted with their surface perpendicular to the target surface normal and $30 \mathrm{~mm}$ distant from the focal spot. The resulting films were analyzed by Raman spectroscopy (Renishaw 2000 system, equipped with a $514.5 \mathrm{~nm}$ laser) and by scanning electron microscopy (SEM) (JEOL 6300LV).
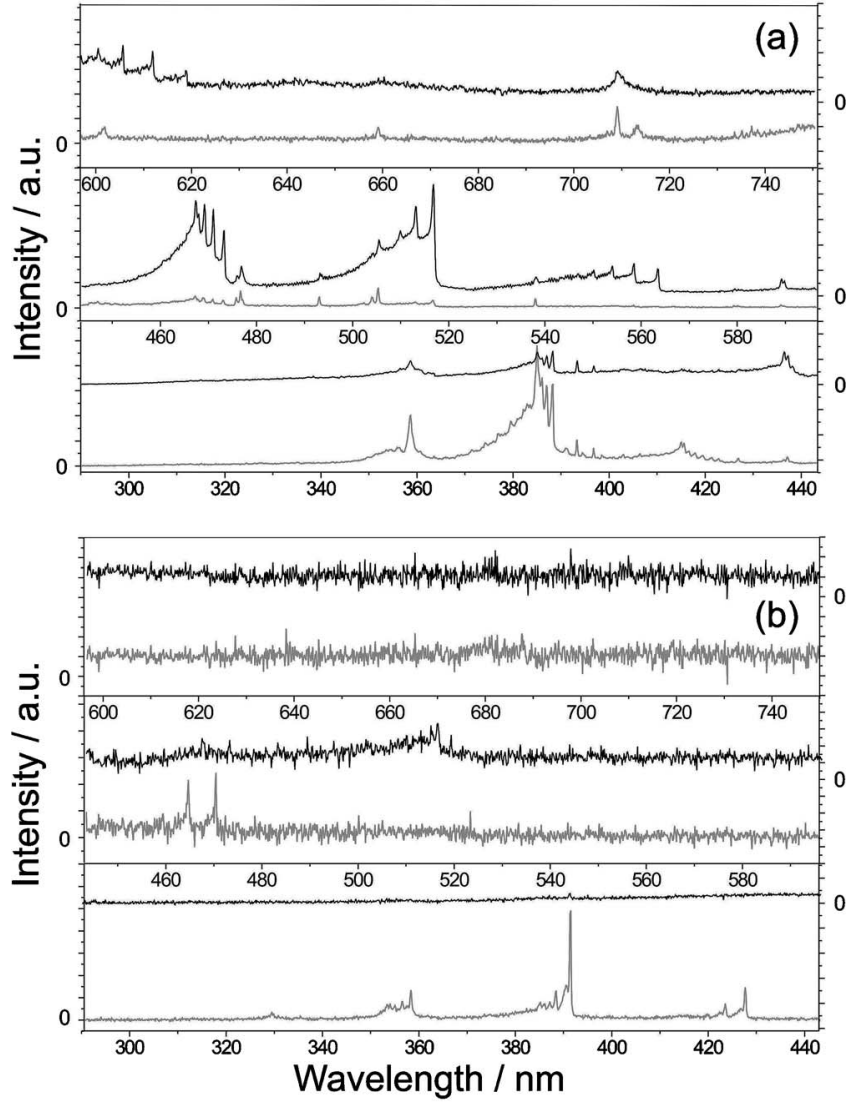

FIG. 2. Wavelength-dispersed spectra (in the range of $290-750 \mathrm{~nm}$ ) of the plume emission accompanying $248 \mathrm{~nm}$ PLA of graphite in $\mathrm{N}_{2}$ using (a) nanosecond and (b) femtosecond laser pulses. The emission is captured using a $1 \mu$ s time gate, delayed by $t=120 \mathrm{~ns}$ from the start of the laser excitation, and accumulated over 80 laser shots. The upper (right axis) and lower (left axis) traces in each panel show spectra recorded at $x=0$ and $5 \mathrm{~mm}$, respectively.

\section{RESULTS}

\section{Wavelength- and spatially resolved OES}

Figure 2 shows optical emission spectra from PLA of graphite in 20 mTorr of $\mathrm{N}_{2}$ using (a) $15 \mathrm{~ns}$ and (b) $450 \mathrm{fs}$ laser pulse durations, recorded over the wavelength range of $290-750 \mathrm{~nm}$. Signal was collected using arrangement (A), with a long $(\delta t \sim 1 \mu \mathrm{s})$ time gate that opened at $t \sim 120 \mathrm{~ns}$ after the instant the laser pulse was incident on the target. The displayed spectra were each collected by accumulating for 80 laser shots while viewing close to the laser/target interaction volume (i.e., at $x=0 \mathrm{~mm}$, upper trace in each panel) and at $x=5 \mathrm{~mm}$ (lower trace). No attempt has been made to correct for any wavelength dependence in the detection sensitivity. The quasicontinuous rising signal at long wavelengths in the $x \sim 5 \mathrm{~mm}$ spectrum in Fig. 2(a) is part of the strong $385 \mathrm{~nm}$ band appearing in second order. The spectrum obtained when viewing close to the target [Fig. 2(a)] displays strong $\mathrm{C}_{2}\left(d^{3} \Pi_{g}-a^{3} \Pi_{u}\right)$ Swan band emission (the strongest, Franck-Condon diagonal, band is centered at $\sim 515 \mathrm{~nm}$ ), several sharp lines due to electronically excited neutral carbon $(\mathrm{C} \mathrm{I})$ atoms and $\mathrm{C}^{+}(\mathrm{C}$ II $)$ ions, ${ }^{8}$ and relatively weak emission from electronically excited $\mathrm{CN}(B)$ radicals at $\sim 385 \mathrm{~nm} . \mathrm{C}_{2}(d-a)$ emission is still discernible in the OES recorded at $x=5 \mathrm{~mm}$ [lower trace in each panel of Fig. 2(a)], 
but this spectrum is dominated by the $\mathrm{CN}\left(B^{2} \Sigma^{+}-X^{2} \Sigma^{+}\right)$ emission that is characteristic of many carbon plasmas in the presence of active nitrogen. Previous studies of $248 \mathrm{~nm}$ PLA of graphite, in vacuum, have revealed $\mathrm{C}_{2}(d-a)$ emissionbut only in the region immediately adjacent to the target surface. ${ }^{8}$ Formation of emitting $\mathrm{C}_{2}$ species is usually considered to involve gas phase collisional processes, in the highpressure and high-temperature region near the target. Introduction of a background gas, as here, and in companion studies of the effect of Ar additions, ${ }^{19}$ should enhance the probability of three-body recombination and/or collisional excitation processes within the plume-consistent with our observation of more intense and extensive $\mathrm{C}_{2}(d-a)$ emission than in the case of PLA in vacuum.

Figures 3(a) and 3(b) compare observed $\mathrm{C}_{2}(d-a)$ and $\mathrm{CN}(B-X)$ spectra (measured at $x=0$ and $5 \mathrm{~mm}$, respectively) with band contours simulated using known spectroscopic constants for the $d^{3} \Pi_{g}$ and $a^{3} \Pi_{u}$ states of $\mathrm{C}_{2},{ }^{20}$ the $B^{2} \Sigma^{+}$ and $X^{2} \Sigma^{+}$states of $\mathrm{CN},{ }^{20,21}$ and the spectral simulation program PGOPHER. ${ }^{22}$ The simulation program assumes Boltzmann vibrational and rotational state population distributions in the emitting species, but allows the option that these distributions have different characteristic temperatures $T_{\mathrm{vib}}$ and $T_{\text {rot }}$. The best-fit temperature values so derived are all hot. For $\mathrm{C}_{2}(d)$, the best-fit (by eye) simulation gives $T_{\text {vib }}$ $\sim 10000 \mathrm{~K}$ and $T_{\text {rot }} \sim 8000 \mathrm{~K}$, while for $\mathrm{CN}(B)$ we find $T_{\mathrm{vib}} \sim 15000 \mathrm{~K}$ and $T_{\mathrm{rot}} \sim 12000 \mathrm{~K}$. These "temperatures" should be regarded as indicative only. The $\mathrm{CN}(B-X)$ simulations overestimate the width of the highest $\left(v^{\prime}, v^{\prime \prime}\right)$ transitions [e.g., the $(13,13)$ feature at $390.6 \mathrm{~nm}$ ]; a better description of the $\mathrm{CN}(B)$ population distribution could probably be obtained using a $T_{\text {rot }}$ function that decreases with increasing $v^{\prime}$. Comparable levels of internal excitation in the $\mathrm{C}_{2}(d)$ and $\mathrm{CN}(B)$ products arising in the UV PLA of graphite ablation in low background pressures of $\mathrm{N}_{2}$ have been deduced previously; ${ }^{23}$ the implied average internal (i.e., vibrational plus rotational) energies amount to $\sim 1$ and $\sim 2 \mathrm{eV}$, respectively.

The spectra displayed in Fig. 2(b) were obtained using $450 \mathrm{fs}$ laser pulses. The overall emission intensity is much lower than in the nanosecond PLA experiment-reflecting, in part at least, the lower incident pulse energy-and consistent with the observed smaller material ejection rate per pulse. ${ }^{8} \mathrm{C}_{2}(d-a) \Delta v=0$ emissions are evident at $\sim 515 \mathrm{~nm}$ when viewing at $x=0$, but much less strongly than in the case of nanosecond PLA. Weak emission attributable to the $\mathrm{N}_{2}^{+}\left(B^{2} \Sigma_{u}{ }^{+}-X^{2} \Sigma_{g}{ }^{+}\right)$system-the most prominent $v^{\prime}=0$ $\rightarrow v^{\prime \prime}=0$ transition appears at $\sim 391 \mathrm{~nm}-$ is also identifiable. This latter emission dominates the OES measured at $x$ $=5 \mathrm{~mm}$, which also contains less intense emission from $\mathrm{CN}(B)$ radicals and two sharp "atomiclike" features at 464.8 and $470.6 \mathrm{~nm}$. These match reasonably with documented transitions of both $\mathrm{C}^{2+}$ and $\mathrm{N}^{+}$ions. The former is most plausibly assigned to the $3 p ;{ }^{3} P^{\mathrm{o}}-3 s ;{ }^{3} S$ transition of $\mathrm{C}^{2+}$, which appeared strongly at short $x$ in the corresponding OES following femtosecond PLA of graphite in vacuum, ${ }^{8}$ although an alternative assignment in terms of the spin-orbit split $2 s^{2} 2 p\left[{ }^{2} P^{0}\right] 3 p ;{ }^{3} P-2 s^{2} 2 p\left[{ }^{2} P^{0}\right] 3 s ;{ }^{3} P^{\mathrm{o}}$ transition of $\mathrm{N}^{+}$ cannot be completely excluded. The assignment of the latter

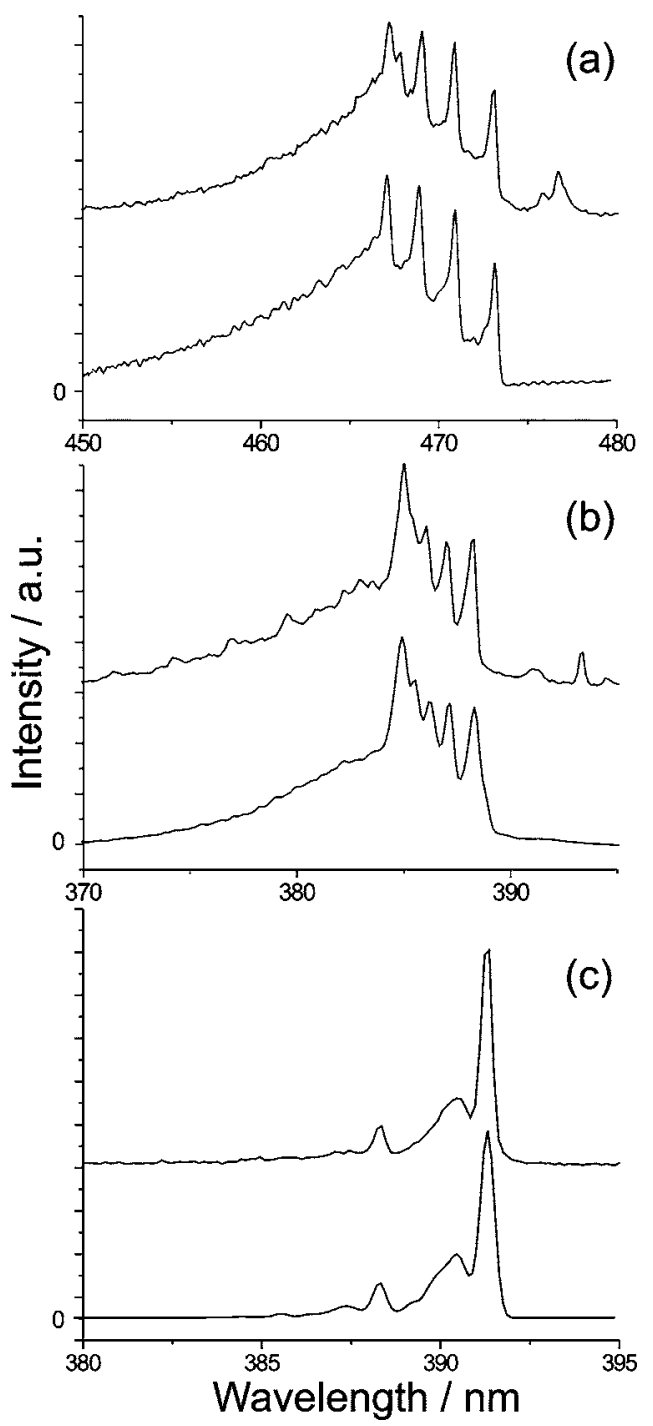

FIG. 3. Observed (upper) and simulated (lower) band contours of (a) $\mathrm{C}_{2}(d-a) \Delta v=-1$ sequence (viewing at $x=0 \mathrm{~mm}$ ) and (b) $\mathrm{CN}(B-X) \Delta v=0$ progression (at $x=5 \mathrm{~mm}$ ) following nanosecond $248 \mathrm{~nm}$ PLA of graphite in $\mathrm{N}_{2}$. The spectral simulations were derived using PGOPHER (see Ref. 22), literature values for the appropriate spectroscopic constants (see Refs. 20 and 21), and vibrational and rotational temperatures and transition linewidths (assumed Gaussian) as follows: (a) $T_{\text {vib }}=10000 \mathrm{~K}, T_{\text {rot }}=8000 \mathrm{~K}$, $\Delta \widetilde{v}=20 \mathrm{~cm}^{-1}$; (b) $T_{\mathrm{vib}} \sim 15000 \mathrm{~K}, T_{\text {rot }} \sim 12000 \mathrm{~K}, \Delta \widetilde{v}=24 \mathrm{~cm}^{-1}$. (c) shows observed (upper) and simulated (lower) band contours of the $\mathrm{N}_{2}{ }^{+}(B-X)$ $\Delta v=0$ sequence (at $x=7 \mathrm{~mm}$ ) following femtosecond $248 \mathrm{~nm}$ PLA of graphite in $\mathrm{N}_{2}$. The best-fit parameters for this simulation are $T_{\mathrm{vib}}$ $=4000 \mathrm{~K}, T_{\text {rot }}=350 \mathrm{~K}$, and $\Delta \widetilde{v}=24 \mathrm{~cm}^{-1}$. Peaks at $\sim 476 \mathrm{~nm}$ in (a) and at $394.2 \mathrm{~nm}$ in (b) are associated with $\mathrm{C}^{*}$ emissions.

emission is even less clear-cut, but might be associated with the $2 s^{2} 2 p\left[{ }^{2} P^{\mathrm{o}}\right] 3 d ;{ }^{1} P^{\mathrm{o}}-2 s^{2} 2 p\left[{ }^{2} P^{0}\right] 3 p ;{ }^{3} S$ transition of $\mathrm{N}^{+}$at $470.9 \mathrm{~nm}$. Other C III emissions (from more highly excited states of $\mathrm{C}^{2+}$ ) as well as a number of $\mathrm{C}$ II emissions were observed in the previous femtosecond PLA studies of graphite at $248 \mathrm{~nm}$ in vacuum. ${ }^{8}$ None of these other emissions were identified in the present work, however. [We note that the traditional signature of electronically excited $\mathrm{C}^{+}$ions, a line at $426.8 \mathrm{~nm}$, could not be used in the present work because of spectral overlap from the (dominant) $\mathrm{N}_{2}{ }^{+}(B-X)$ $\Delta v=+1$ sequence; however, the band contour simulations suggest that such $\mathrm{C}^{+}$emission makes negligible contribution to the integrated (over $1 \mu \mathrm{s}$ ) total emission intensity at this wavelength in the $x=5 \mathrm{~mm}$ spectrum.] 


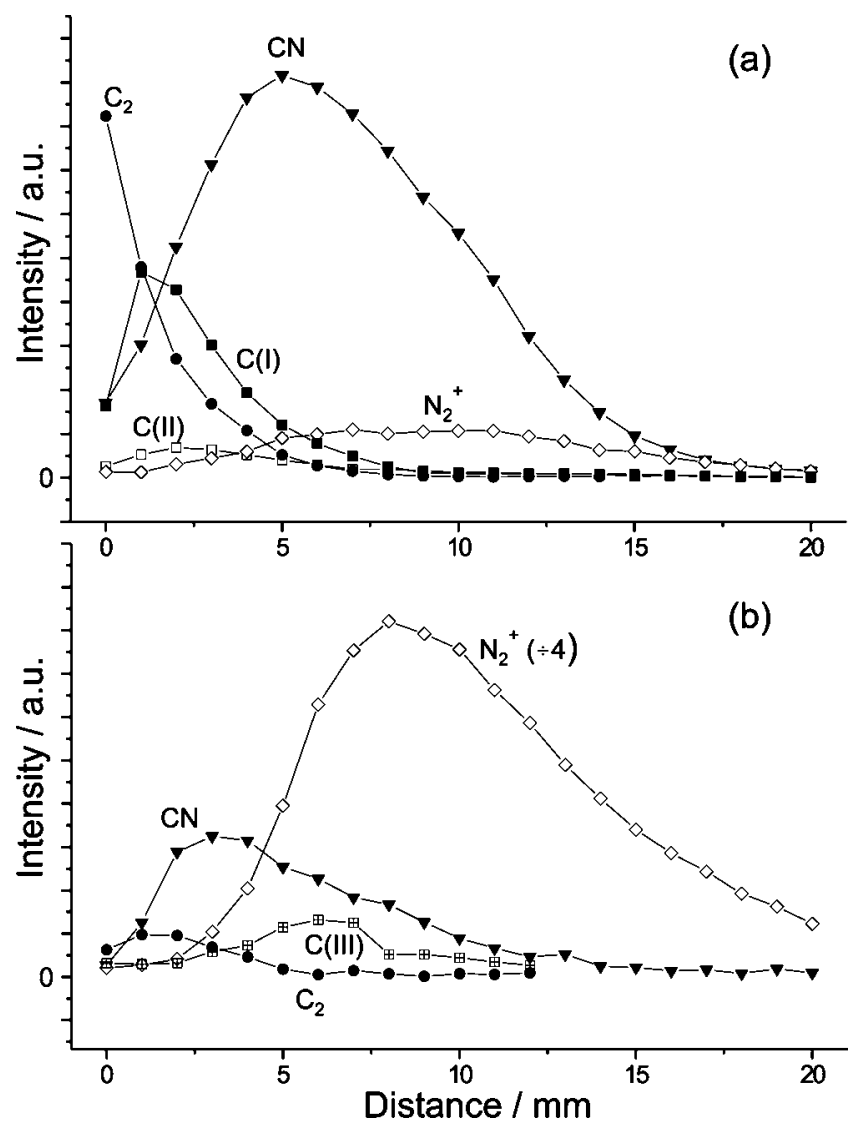

FIG. 4. Plots showing the $x$ dependences of the peak emission intensities associated with $\mathrm{C}_{2}, \mathrm{C} \mathrm{I}, \mathrm{C} \mathrm{II}, \mathrm{C}$ III, CN, and $\mathrm{N}_{2}{ }^{+}$species following $248 \mathrm{~nm}$ PLA of graphite in $\mathrm{N}_{2}$ using (a) nanosecond and (b) femtosecond laser pulses. The plotted relative intensities are as observed, except in the case of $\mathrm{N}_{2}{ }^{+}$signal in (b) which has been reduced fourfold so that the $x$-dependent trends of the other emitters can be seen more clearly. These relative scalings involve considerable arbitrariness, since they depend on many factors, including the average excited-state populations during the $1 \mu \mathrm{s}$ observation time, the radiative and quenching properties of the various emitters, the choice of wavelength for monitoring any particular emitter, and the wavelength-dependent spectrometer response function.

Figure 4 depicts the $x$ dependence of selected emission features obtained by analyzing OES like those displayed in Fig. 2, taken at many different $x$ values. Figure 4(a) shows data for the case of nanosecond excitation. Emissions attributable to pure carbon species, i.e., $\mathrm{C}$ I, C II, and $\mathrm{C}_{2}$ [monitored via the $4 p ;{ }^{3} P-3 s ;{ }^{3} P^{\mathrm{o}}$ line at $476.7 \mathrm{~nm}$, the $4 f ;{ }^{2} F^{\mathrm{o}}-3 d ;{ }^{2} D$ transition at $426.8 \mathrm{~nm}$, and the $d-a(0,0)$ peak at $516.6 \mathrm{~nm}$, respectively], are all seen to maximize at $x \sim 0$. The most intense feature is the $\mathrm{CN}(B-X) \Delta v=0$ sequence, centered (and measured) at $385 \mathrm{~nm}$, which appears to peak at $x \sim 5 \mathrm{~mm}$. The signal associated with $\mathrm{N}_{2}{ }^{+}$shows the weakest $x$ dependence, maximizing at $x \sim 9 \mathrm{~mm}$ and declining very gradually thereafter. Clearly, it is necessary to exercise some caution when assessing rather weak $\mathrm{N}_{2}{ }^{+}(B-X)$ signals in the presence of stronger (and overlapping) $\mathrm{CN}(B-X)$ emissions; trends for the former species were deduced from analysis of emissions at $\sim 391$ and $\sim 356 \mathrm{~nm}$.

Figure 4(b) shows the corresponding emission intensity versus $x$ dependences obtained by analyzing spectra of the plume emission during femtosecond PLA. One difference is immediately apparent: emission from $\mathrm{N}_{2}^{+}(B)$ ions, rather

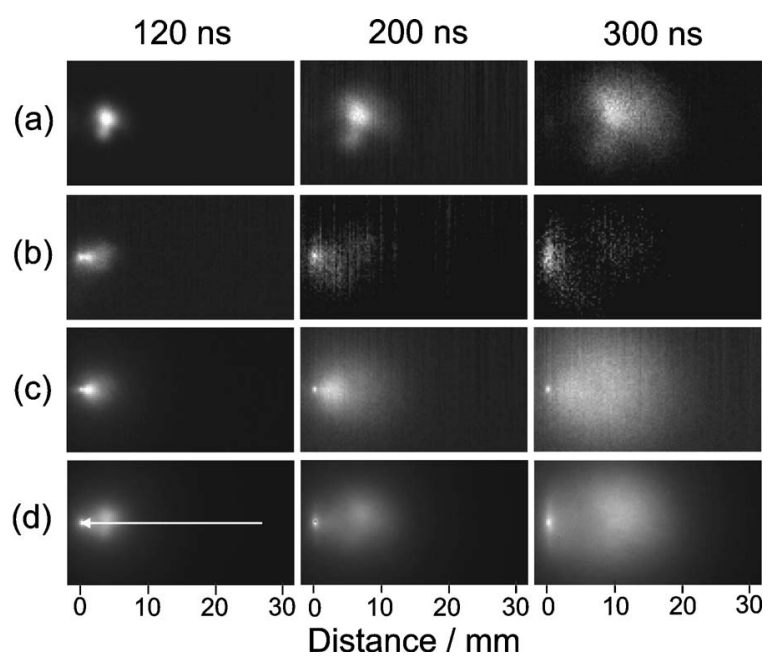

FIG. 5. i-CCD images of wavelength-selected components of the OES [(a)(c)] and the total OES (d) from the nanosecond $248 \mathrm{~nm}$ PLA of graphite in $\mathrm{N}_{2}$. Each image was recorded using a $20 \mathrm{~ns}$ intensifier time gate, delayed by $t=120,200$, and $300 \mathrm{~ns}$ relative to the laser pulse (left-hand center, and right hand columns, respectively), and is displayed using the full range of the available gray scale. Wavelength-selected images were taken using (a) a $426 \mathrm{~nm}$ narrow bandpass filter, (b) a bandpass filter centered at $520 \mathrm{~nm}$, and (c) a long pass filter that only transmits $\lambda>780 \mathrm{~nm}$. The white arrow shown in the bottom left-hand panel only, but applicable to all of the displayed images, indicates the projection of the laser propagation axis onto the viewing $(x z)$ plane.

than $\mathrm{CN}(B)$ radicals, dominates the OES at larger $x$. The relative intensities of the $\mathrm{N}_{2}{ }^{+}(B-X)$ signals in Fig. 4(b) have been reduced fourfold to allow clearer observation of the other species trends. The $\mathrm{N}_{2}{ }^{+}(B-X)$ emission intensity peaks at $x \sim 8 \mathrm{~mm}$ (similar to the nanosecond case) and is still the dominant emission at $x \sim 20 \mathrm{~mm}$. Figure 3(c) shows simulations of the observed band contours, using PGOPHER (Ref. 22) together with known spectroscopic constants for the $B$ and $X$ states. $^{20}$ The temperatures so derived, $T_{\text {vib }} \sim 4000 \mathrm{~K}$ and $T_{\text {rot }} \sim 350 \mathrm{~K}$, are strikingly different from those obtained for the $\mathrm{C}_{2}(d)$ and $\mathrm{CN}(B)$ emitters observed in the case of nanosecond PLA. Emission at $464.7 \mathrm{~nm}$-due to electronically excited $\mathrm{C}^{2+}$ ions-peaks at $x \sim 6 \mathrm{~mm}$. Emission from any other carbon-based species at such large $x$ is negligible in comparison. Emission from $\mathrm{CN}(B)$ radicals peaks at $x$ $\sim 3 \mathrm{~mm}$, i.e., $\sim 2 \mathrm{~mm}$ closer to the laser-target interaction region than in the nanosecond case, and thereafter declines comparatively slowly. The weak $\mathrm{C}_{2}(d-a)$ emission peaks at even smaller $x$-as in the nanosecond case.

\section{Spatially and temporally resolved images of selected optical emissions}

Spatially and wavelength-resolved data of the kind shown in Fig. 2, analyzed to yield the plots in Fig. 4, are recorded using a $\sim 1 \mu$ s time gate. Such spectra allow unambiguous species identification, and provide time-integrated information about the spatial distributions of the various emitting species. Time-resolved measurements of the kind shown in Figs. 5 and 6 have the potential to provide alternative and additional insights into these spatial distributions and the formation and destruction mechanisms of the emitting species. Figure 5 shows CCD images of the plume ac- 


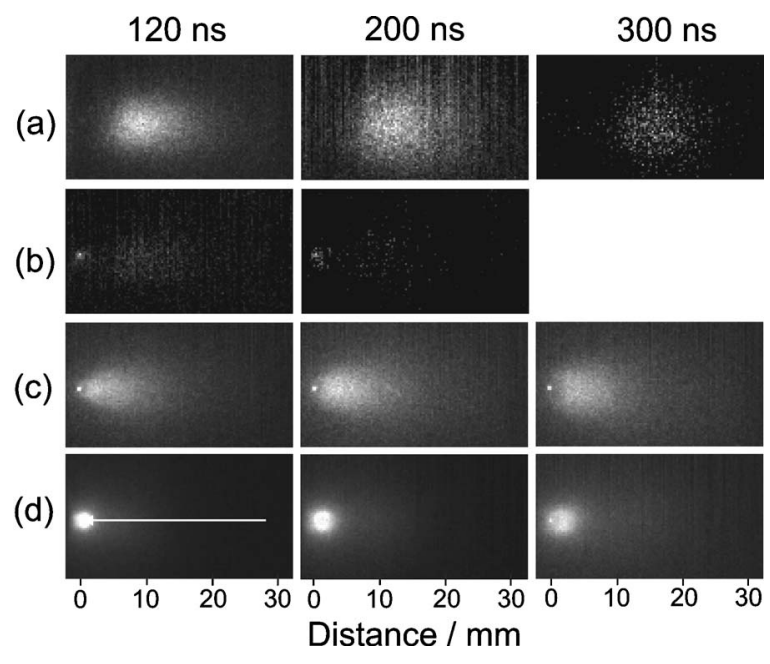

FIG. 6. i-CCD images of wavelength-selected components of the OES [(a)(c)] and the total OES (d) from the femtosecond $248 \mathrm{~nm}$ PLA of graphite in $\mathrm{N}_{2}$. As in Fig. 5, each image was recorded using a $20 \mathrm{~ns}$ intensifier time gate, delayed by $t=120,200$, and $300 \mathrm{~ns}$ relative to the laser pulse (left-hand center, and right-hand columns, respectively), and is displayed using the full range of the available gray scale. Wavelength-selected images were taken using (a) a $426 \mathrm{~nm}$ narrow bandpass filter, (b) a bandpass filter centered at $520 \mathrm{~nm}$, and (c) a long pass filter that only transmits $\lambda>780 \mathrm{~nm}$. The white arrow shown in the bottom left hand panel only, but applicable to all of the displayed images, indicates the projection of the laser propagation axis onto the viewing $(x z)$ plane.

companying nanosecond PLA using arrangement (B), and a very short $(\delta t=20 \mathrm{~ns})$ intensifier gate width delayed by $t$ $=120,200$, and 300 ns (left-hand, center, and right-hand columns, respectively). Each is a projection of the threedimensional (3D) plume emission squashed into the $x$ (horizontal) $-z$ (vertical) plane. The white arrow (shown in the bottom left-hand panel only, but applicable to all of the displayed images) shows the projection of the laser propagation axis onto the viewing plane. Images in rows (a) and (b) were taken through narrow bandpass filters centered at 426 and at $520 \mathrm{~nm}$, respectively. Images in row (c) were recorded through a long pass filter that only transmits wavelengths $\lambda$ $>780 \mathrm{~nm}$, while those in row (d) are of the total (i.e., unfiltered) emission. Clearly, the $520 \mathrm{~nm}$ emission is only observed close to the target surface, and expands much more slowly than the other components. Figure 6 shows the corresponding set of images obtained in the case of femtosecond PLA.

Discussion of the carriers of these various emissions is involved, and thus reserved until later, pending analysis of the intensity profiles to allow estimation of the propagation velocities $\langle v\rangle$ of the various emitters. Figure 7 shows representative intensity $(I)$ vs $x$ cuts along the center axis of the (a) $426 \mathrm{~nm}$, (b) $520 \mathrm{~nm}$, and (c) $\lambda>780 \mathrm{~nm}$ images resulting from nanosecond PLA, recorded at four different delays, $t$. Each such profile was fitted using one or more skewed Gaussian functions, mainly to determine the position of peak emission intensity, $x_{\text {peak }}$. Figure 8 shows corresponding $I$ vs $x$ plots derived from the (a) $426 \mathrm{~nm}$, (b) $\lambda>780 \mathrm{~nm}$, and (c) total OES images resulting from femtosecond PLA, again recorded at four different delays, $t$, while Figs. 9(a) and 9(b) show plots of the derived $x_{\text {peak }}$ values versus $t$ from images recorded in both the nanosecond and femtosecond PLA ex-

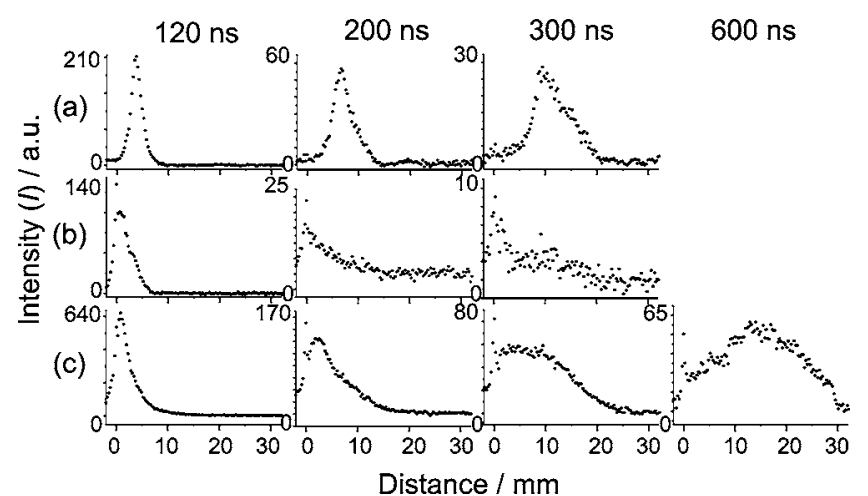

FIG. 7. Intensity $(I)$ vs distance $(x)$ profiles derived from wavelengthselected i-CCD images of the plume arising from the nanosecond $248 \mathrm{~nm}$ PLA of graphite in $\mathrm{N}_{2}$, recorded at $t=120,200,300$, and $600 \mathrm{~ns}$. The row labels (a), (b), and (c) display images taken using (a) a $426 \mathrm{~nm}$ narrow bandpass filter, (b) a bandpass filter centered at $520 \mathrm{~nm}$, and (c) a long pass filter that only transmits $\lambda>780 \mathrm{~nm}$, respectively. Peak values $\left(x_{\text {peak }}\right)$ have been obtained by fitting skewed Gaussian functions to these profiles.

periments. The slopes of such plots provide measures of the propagation velocities of the various emissions, though we recognize that plume expansion in the $x z$ plane means that any analysis based solely on a slice along the center $(z=0)$ axis will tend to underestimate the relative yield of fast material and thus $\langle v\rangle$.

We focus first on the emissions accompanying nanosecond PLA. The $x_{\text {peak }}$ values for the total emission intensity profiles are denoted by $\boldsymbol{\nabla}$ in Fig. 9(a). A propagation velocity $\langle v\rangle=41.3 \pm 0.6 \mathrm{~km} \mathrm{~s}^{-1}$ along the surface normal can be deduced by a straight line fit to the short distance, early time, observations $\left(x_{\text {peak }} \leqslant 10 \mathrm{~mm}, t \leqslant 280 \mathrm{~ns}\right)$; the available data thereafter are more widely spaced in $t$ but suggest that the expansion of the peak of the distribution of emitting species stagnates (until $t \sim 1250 \mathrm{~ns}$ ) and then accelerates again. As discussed more fully later, the apparent behavior at larger $x$ is complicated by the effects of species being reflected from the substrate, the front surface of which is at $x=30 \mathrm{~mm}$. The $426 \mathrm{~nm}$ emission $(\square)$ data yields a similar propagation velocity $\langle v\rangle=36.4 \pm 0.5 \mathrm{~km} \mathrm{~s}^{-1}$, but has faded to invisibility by $t \sim 380 \mathrm{~ns}$. The $\lambda>780 \mathrm{~nm}$ emission (ם) shows a lower initial slope $\left(\langle v\rangle=20.8 \pm 1.2 \mathrm{~km} \mathrm{~s}^{-1}\right)$, but then appears to accel-

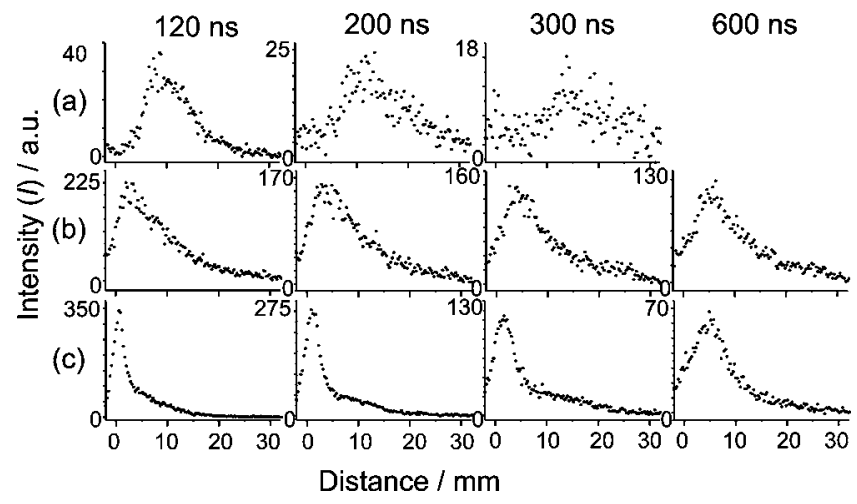

FIG. 8. I vs $x$ profiles derived from wavelength-selected i-CCD images of the plume from femtosecond $248 \mathrm{~nm}$ PLA of graphite in $\mathrm{N}_{2}$, recorded at $t$ $=120,200,300$, and $600 \mathrm{~ns}$ : (a) $426 \mathrm{~nm}$ emission; (b) $\lambda>780 \mathrm{~nm}$ emission, and (c) total OES. Peak values $\left(x_{\text {peak }}\right)$ have been obtained by fitting skewed Gaussian functions to the derived profiles. 


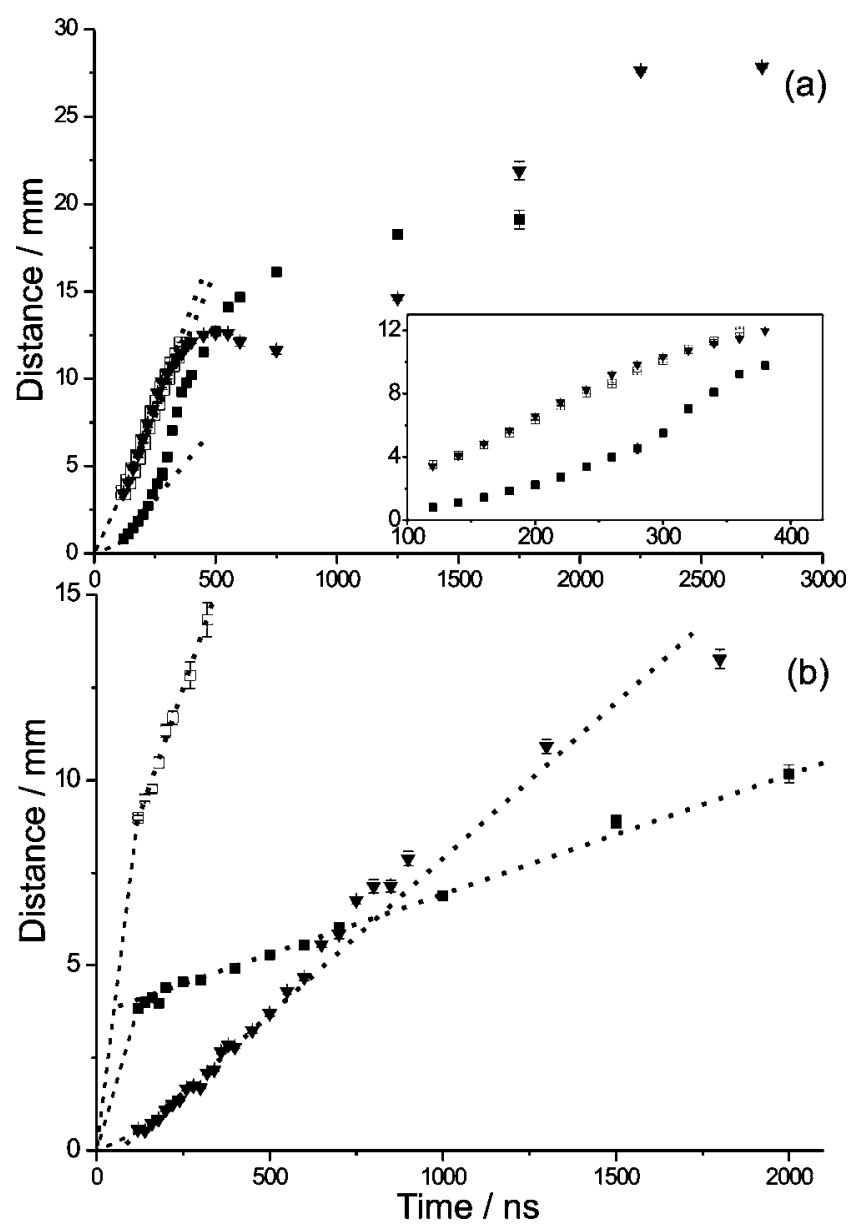

FIG. 9. $x_{\text {peak }}$ vs $t$ plots derived from fitting skewed Gaussian profiles to $I$ vs $x$ plots of the kind illustrated in Figs. 7 and 8 which, in turn, are derived from analysis of time-gated imaging of the plume accompanying (a) nanosecond and (b) femtosecond $248 \mathrm{~nm}$ PLAs of graphite in $\mathrm{N}_{2}$. The gradients of the illustrative lines of best fit (dotted lines) provide propagation velocities for the various emissions, labeled as follows: total OES $(\boldsymbol{\nabla}), 426 \mathrm{~nm}$ emission ( $\square$ ) and $\lambda>780 \mathrm{~nm}$ emission ( $\square$ ). The inset in (a) provides an expanded view of the early time $x_{\text {peak }}$ vs $t$ trends in the case of nanosecond PLA.

erate so that, by $t \sim 400 \mathrm{~ns}$, the average propagation velocity of this emission is similar to that of both the total and the $426 \mathrm{~nm}$ emissions; thereafter, the $\lambda>780 \mathrm{~nm}$ emission also appears to expand much more slowly.

We now try to correlate these data with the wavelengthdispersed spectra of the plume accompanying nanosecond PLA recorded with a long $(1 \mu \mathrm{s})$ time gate. The upper $(x$ $=0)$ spectrum shown in Fig. 2(a) is dominated by emissions from $\mathrm{C}$ atoms, $\mathrm{C}^{+}$ions, and $\mathrm{C}_{2}(d)$ radicals, whereas $\mathrm{CN}(B)$ radicals make the dominant contribution in the lower $(x$ $=5 \mathrm{~mm}$ ) spectrum. Clearly, the species that show strongly in these spectra must be those that make the greatest contribution when integrating throughout the $1 \mu$ s viewing window, but additional information is needed if we are to tell whether any given feature is from a relatively intense but short-lived emission or from a weaker but persistent emission. The timegated images provide some of the necessary insights. Images recorded through the $520 \mathrm{~nm}$ bandpass filter are unarguably associated with $\mathrm{C}_{2}(d-a)$ emission. This emission is most intense at small $x$ and at short $t$, but decays rapidly at longer time delays. The wavelength-dispersed spectra [Fig. 2(a)], the peak intensity versus distance dependence derived there from [Fig. 4(a)], and the time-gated images all indicate that $\mathrm{C}_{2}(d)$ species are concentrated close to the laser-target interaction region. The localized contributions from $\mathrm{C}_{2}(d-a)$ emission are also recognizable (at $x \sim 0$ ) in the images of total emission (bottom row in Fig. 5).

The total emission images reveal the presence of faster components in the plume. Figure 4(a) suggests that much of the imaged total emission must be associated with $\mathrm{CN}(B)$ radicals, but none of the bandpass filters employed are appropriate for isolating $\mathrm{CN}(B-X)$ emission. $\mathrm{CN}$ radicals in their lower-lying $A^{2} \Pi$ state, if present, would emit at $\lambda$ $>780 \mathrm{~nm},{ }^{20}$ however. So, too, would a range of $\mathrm{C}_{\mathrm{I}}$ transitions-which have been observed in previous studies of graphite ablation, in vacuum, in this apparatus. ${ }^{8}$ Thus, we propose two distinct contributions to the time-gated images of $\lambda>780 \mathrm{~nm}$ emission; C I emissions, which are most important at small $x$ and $t$, and $\mathrm{CN}(A-X)$ emission which becomes dominant as $x, t$, and the number of collisions between ablated carbon species and background $\mathrm{N}_{2}$ increases. Such an interpretation is supported by the $I$ vs $x$ plots derived from the $\lambda>780 \mathrm{~nm}$ images [Fig. 7(c)], which we interpret in terms of a slow component, that appears strongly at early time but fades as $t$ increases, and a less intense but more persistent faster (i.e., larger $x$ ) component that dominates the later time images. This progressive switch in the identity of the dominant emitter accounts for the nonlinearity in the early time part of the $x$ vs $t$ plot derived from the $\lambda$ $>780 \mathrm{~nm}$ images [Fig. 9(a)]. Previous studies of C I emissions arising in the $248 \mathrm{~nm}$ PLA of graphite, in vacuum, in this same apparatus, yielded initial propagation velocities $\langle v\rangle \sim 17 \mathrm{~km} \mathrm{~s}^{-1}$ (Ref. 8) - similar to the early time slope of the $\lambda>780 \mathrm{~nm}$ emission in Fig. 9(a). Reasons why the $\mathrm{CN}(A)$ emitters - that are proposed to develop with increasing $t$ - should exhibit faster expansion velocities than the $\mathrm{C}$ I emissions are discussed later. Nonetheless, the accumulated evidence at this stage suggests that $\mathrm{CN}(B)$ and $\mathrm{CN}(A)$ radicals both contribute to the time-gated total emission images, that the latter make a major contribution to the $\lambda>780 \mathrm{~nm}$ images, and that both emissions propagate with similar velocities-i.e., $\sim 40 \mathrm{~km} \mathrm{~s}^{-1}$ up to $t \sim 400 \mathrm{~ns}$ and more slowly thereafter.

We now consider the images obtained viewing the plume accompanying nanosecond PLA through the $426 \mathrm{~nm}$ bandpass filter. This filter will transmit $\mathrm{C}$ II emission associated with the $\mathrm{C}^{+}\left(4 f ;{ }^{2} F^{\mathrm{o}}-3 d ;{ }^{2} D\right)$ transition at $426.7 \mathrm{~nm}$ - which showed strongly in studies of the $248 \mathrm{~nm}$ PLA of graphite in vacuum $^{8}$ and in low pressures of Ar. ${ }^{19}$ It would also transmit $\mathrm{N}_{2}{ }^{+}(B-X) \Delta v=+1$ emission-if present. $\mathrm{N}_{2}{ }^{+}(B-X)$ emission is clearly evident in the femtosecond PLA studies and, as Fig. 2(b) illustrates, the Franck-Condon factors associated with the $\mathrm{N}_{2}{ }^{+}(B-X)$ transition ensure that the $\Delta v=0$ sequence will always be much more intense than the $\Delta v=+1$ sequence. Wavelength-dispersed spectra obtained following nanosecond PLA of graphite in $\mathrm{N}_{2}$ [Fig. 2(a)] show no obvious $\mathrm{N}_{2}^{+}(B-X)$ emission. Thus we conclude that the $426 \mathrm{~nm}$ images shown in Fig. 5(a) map the propagation of emitting $\mathrm{C}^{+}$ions. This emission has died out by $t \sim 400 \mathrm{~ns}$, but the deduced propagation velocity up to that time, $\langle v\rangle$ 
$=36.4 \pm 0.5 \mathrm{~km} \mathrm{~s}^{-1}$, is similar to that found for the $\mathrm{CN}$ emissions. The deduction that the speeds of the emitting $\mathrm{C}^{+}$ions and $\mathrm{CN}$ radicals are similar, and faster than those of the emitting $\mathrm{C}$ neutrals, may offer clues about the collisional processes responsible for the observed $\mathrm{CN}$ emissions.

Analysis of wavelength-dispersed spectra of the plume emission accompanying femtosecond PLA such as those shown in Fig. 2(b) revealed the presence of $\mathrm{C}_{2}(d)$ radicals at small $x, \mathrm{CN}(B)$ radicals-localized at smaller $x$ than in the case of nanosecond PLA - and substantial emission from $\mathrm{N}_{2}{ }^{+}(B)$ ions. The short (20 ns)-time-gated images shown in Fig. 6 provide complementary insights. As in the nanosecond PLA studies, the weak, rapidly decaying emission imaged through the $520 \mathrm{~nm}$ bandpass filter and localized close to the laser-target interaction volume is attributable to $\mathrm{C}_{2}(d)$ radicals. As discussed above, the $426 \mathrm{~nm}$ bandpass filter can transmit both $\mathrm{C}^{+}\left(4 f ;{ }^{2} F^{\mathrm{o}}-3 d ;{ }^{2} D\right)$ and $\mathrm{N}_{2}{ }^{+}(B-X) \Delta v=+1$ emissions. The latter dominate the (time-integrated) wavelength-dispersed emission spectra, but the deduced speed of the $426 \mathrm{~nm}$ emitters in the short $t$ images shown in Fig. 6(a) suggests that, as in the nanosecond case, excited $\mathrm{C}^{+}$ ions are the main contributions to the early time emission at this wavelength. As Fig. 9(b) shows, the average speed of these emitting $\mathrm{C}^{+}$ions must be $\sim 75 \mathrm{~km} \mathrm{~s}^{-1}$ in the early $(t$ $\leqslant 120 \mathrm{~ns}$ ), preobservation stages of the expansion; the bestfit gradient of the $x_{\text {peak }}$ vs $t$ plot in the observation range $120 \leqslant t \leqslant 350 \mathrm{~ns}$ is $\langle v\rangle=28 \pm 2 \mathrm{~km} \mathrm{~s}^{-1}$. These values, and the trend in the $x_{\text {peak }}$ vs $t$ plot, are reminiscent of those found in the case of femtosecond $248 \mathrm{~nm}$ PLA of graphite in vacuum. ${ }^{8}$ The $\lambda>780 \mathrm{~nm}$ images [Fig. 6(c)] also reveal emission from a spread of $x$ values at early $t$. As in the nanosecond PLA experiments, we associate this with $\mathrm{CN}(A)$ fragments which we assume to be formed in parallel with the $\mathrm{CN}(B)$ fragments identified in the wavelength-dispersed spectra recorded at small $x$. What is also evident from the images in Fig. 6(c), however, and clearer still from the gradient of the corresponding $x_{\text {peak }}$ vs $t$ plot [Fig. 9(b)], is that the subsequent expansion of this emission is slow, $\langle v\rangle$ $=3.4 \pm 0.6 \mathrm{~km} \mathrm{~s}^{-1}$.

One other notable aspect of Fig. 6 is the dissimilarity between the images of the total OES and those of the various wavelength-selected emissions recorded with the same time gate. Clearly, the dominant contributor to the total OES propagates at lower velocity than the species responsible for the early time $426 \mathrm{~nm}$ and $\lambda>780 \mathrm{~nm}$ emissions [i.e., $\mathrm{C}^{+}$ ions and $\mathrm{CN}(A)$ radicals, respectively]. Such faster emission is discernable in the total OES images shown in Fig. 6(d), and can be seen more clearly from the corresponding $I$ vs $x$ plots shown in Fig. 8(c). Given the dominance of $\mathrm{N}_{2}{ }^{+}(B-X)$ emission in the wavelength-resolved spectra recorded at $x$ $>0$ [see Fig. 4(b), and the $x=5 \mathrm{~mm}$ data shown in Fig. 2(b)], it is reasonable to assume that this emission is the dominant contributor to the total OES images also. The gradient of the $x_{\text {peak }}$ vs $t$ plot of this emission [ $\boldsymbol{\nabla}$ in Fig. 9(b)] yields an apparent propagation velocity $\langle v\rangle=9.7 \pm 0.2 \mathrm{~km} \mathrm{~s}^{-1}$.

\section{Analysis of deposited $\mathrm{CN}_{x}$ films}

Material coating was not the major thrust of the present work. Nonetheless, it was clearly important to establish that

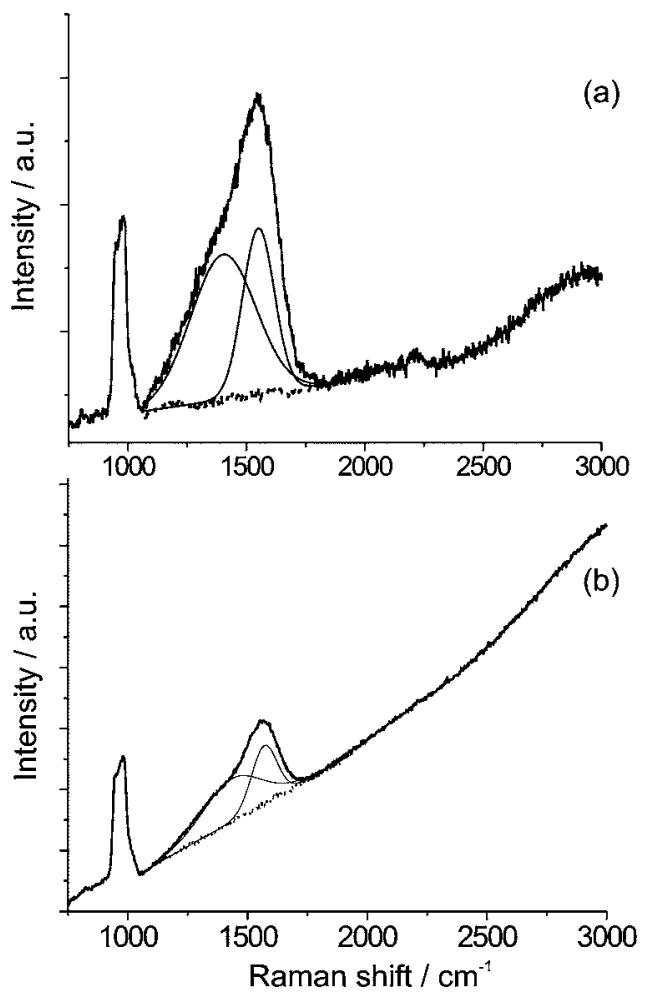

FIG. 10. Raman spectra (514.5 nm excitation wavelength) of $\mathrm{CN}_{x}$ films deposited on Si by (a) nanosecond and (b) femtosecond PLAs of graphite in $\mathrm{N}_{2}$. Also shown are deconvolutions of the $1050-1800 \mathrm{~cm}^{-1}$ feature in terms of two independent Gaussian functions (solid curves) after subtraction of a (linear) background contribution attributable to photoluminescence. Residuals of the fit are shown by dotted lines. The strong peak centered at $\sim 950 \mathrm{~cm}^{-1}$ is due to the underlying Si substrate.

the process conditions employed did yield $\mathrm{CN}_{x}$ films. Thus films were grown by both nanosecond and femtosecond PLA, on Si substrates maintained at room temperature and mounted at $x=30 \mathrm{~mm}$ with their front surface perpendicular to the surface normal, and then analyzed visually, by SEM and by Raman spectroscopy $(514.5 \mathrm{~nm}$ excitation wavelength). SEM showed both sets of films to be smooth and relatively free from particulates. Films grown by nanosecond PLA were significantly thicker, however. For example, $\sim 900$ ns laser pulses were sufficient to produce a visible brown coating, whereas the corresponding film produced using 900 fs pulses could not be distinguished (by eye) from the underlying silicon surface. As Fig. 10 shows, both sets of films showed characteristic $\mathrm{CN}_{x}$ Raman signatures in the $1050-1800 \mathrm{~cm}^{-1}$ region. ${ }^{24}$ These were deconvoluted into contributions associated with the $D$ and $G$ vibrational modes of graphite, ${ }^{25}$ using Gaussian functions as shown in Fig. 10. The $D$ to $G$ band intensity ratio, $I(D) / I(G)$, can provide insights into the extent of clustering induced when $\mathrm{N}$ is incorporated into amorphous carbon films. Higher $I(D) / I(G)$ ratios are considered to imply increased clustering and larger cluster sizes of the $s p^{2}$ phase material. The $I(D) / I(G)$ values derived for the present $\mathrm{CN}_{x}$ thin films are 0.87 and 0.74 , for the case of nanosecond and femtosecond PLA, respectively. These values are both comparable to those found in previous studies of films deposited at room temperature using nanosecond $193 \mathrm{~nm}$ PLA of graphite in 20 mTorr of $\mathrm{N}_{2}(\sim 0.9$, Ref. 24), and much higher than the $\sim 0.15$ values found in 
the $\mathrm{N}_{2}$-free case, e.g., when $\mathrm{N}_{2}$ is replaced by an equivalent background pressure of Ar. ${ }^{19,24}$ Such relatively high $I(D) / I(G)$ values for $\mathrm{CN}_{x}$ thin films deposited at room temperature confirm nitrogen incorporation within the carbon lattice, where it serves to relax some of the constraints associated with nanocrystalline domain formation. ${ }^{24}$ So, too, does the weak feature at $\sim 2225 \mathrm{~cm}^{-1}$ in the spectrum of the film grown by nanosecond PLA. This peak is traditionally attributed to the presence of the nitrile $(\mathrm{C} \equiv \mathrm{N})$ group. Its nonobservation in the Raman spectra of films grown by femtosecond PLA may suggest that the extent of $\mathrm{N}$ incorporation and/or bonding is lower than in the case of films grown by nanosecond PLA-consistent with the somewhat lower $I(D) / I(G)$ ratios observed for such films. However, we also note the comparative weakness of the Raman signal from the $\mathrm{CN}_{x}$ film grown by femtosecond PLA [as compared with the background Si feature at $\sim 950 \mathrm{~cm}^{-1}$ (Fig. 10(b))], reflecting the facts that femtosecond PLA yields thinner films than those grown from nanosecond pulses of the same fluence, and that the amount of material ejected per femtosecond pulse is much smaller.

\section{DISCUSSION}

Film analysis confirms that $\mathrm{CN}_{x}$ films are grown by $248 \mathrm{~nm}$ PLA of graphite in a low pressure of $\mathrm{N}_{2}$, using both nanosecond and femtosecond laser pulse durations, and that nanosecond PLA results in films that are thicker and have a higher degree of $\mathrm{N}$ incorporation. The main focus of the present work, however, is the use of wavelength-, spatially, and temporally resolved OESs to unravel clues as to how $\mathrm{N}$ is incorporated within such films.

Irrespective of the pulse duration, the initial laser-target interaction can only lead to ejection of carbonaceous material, mainly atoms and atomic ions, as well as electrons. As in the case of ablation in vacuum, ${ }^{8}$ or in a low background pressure of $\mathrm{Ar}^{19}$ the most likely route to forming $\mathrm{C}_{2}$ species will involve atom-atom recombination reactions, stabilized by a third body (generally $\mathrm{N}_{2}$ in the present case). Since the $\mathrm{C}$ atom (and ion) density will be highest in the small volume adjacent to the irradiated area on the target surface, the observation that the $\mathrm{C}_{2}(d-a)$ emission intensity peaks at small $x$ and $t$ is unsurprising. Similarly, the observation that the $\mathrm{C}_{2}(d-a)$ emission in the femtosecond PLA study is weaker, and even more spatially localized, accords with the finding that femtosecond PLA causes less material removal per pulse.

Emitting $\mathrm{C}, \mathrm{C}^{+}$, and $\mathrm{C}^{2+}$ species have all been identified in previous UV PLA studies of graphite in vacuum, ${ }^{8,26}$ and in $\mathrm{Ar}^{19}{ }^{19}$ and propagation velocities reported that are similar to those measured in the present work. The natural radiative lifetimes $\tau_{\text {rad }}$ of many of the emitting states of these atomic species are significantly shorter than the times over which their emission is observed, implying the operation of one (or more) mechanism for populating the observed emitting levels, post-ablation. One such mechanism in the case of atomic species is collisionally assisted electron-ion recombination (EIR), whereby $\mathrm{M}^{n+}$ ions and electrons combine to yield $\mathrm{M}^{(n-1)+}$ species in high-lying Rydberg states; these subse- quently relax-collisionally and/or by radiative cascadethrough a manifold of relatively long-lived Rydberg states and eventually pass through the transitions monitored by OES. ${ }^{26}$ Analyses of wavelength-dispersed OES accompanying previous femtosecond PLA studies of graphite in vacuum have indicated a significantly higher degree of ionization within the ejected material than in the case of nanosecond PLA. ${ }^{8}$ Such is expected to be the case here also, but the emissions from most of the excited $\mathrm{C}^{n+}$ species [e.g., the C II emissions imaged in Fig. 6(a)] are presumably too weak, and decay (radiatively or collisionally) too rapidly, to make a discernible contribution to the wavelength-dispersed OES recorded with a long $(1 \mu \mathrm{s})$ time gate.

Of the other observed molecular emitters, $\mathrm{CN}(B)$ and $\mathrm{N}_{2}{ }^{+}(B)$ species both have $\tau_{\text {rad }}$ values [in the range of 60-70 ns (Ref. 20)] that are also much less than the times over which their emission is observed. $\tau_{\text {rad }}$ for the $\mathrm{CN}(A)$ radical is about one order of magnitude longer. ${ }^{20}$ It seems reasonable to assume that $\mathrm{CN}$ formation involves reaction between a carbon-containing species in the ablation plume and a background $\mathrm{N}_{2}$ molecule. However, any detailed interpretation will require more detailed knowledge of the carbon atom and ion, and $\mathrm{C}_{2}$ radical, number density distributions, their respective kinetic-energy distributions, and their respective collision-energy-dependent reaction cross sections with $\mathrm{N}_{2}$ than can be provided simply by OES measurements.

Previous investigations of emitting electronically, vibrationally, and rotationally excited $\mathrm{CN}$ radicals (henceforth represented simply as $\mathrm{CN}^{*}$ if the electronic state is unimportant) formed via PLA of graphite in $\mathrm{N}_{2}$ (Refs. 27-30) have invoked a role for the four-center reaction

$$
\mathrm{C}_{2}+\mathrm{N}_{2} \rightarrow 2 \mathrm{CN},
$$

which is only slightly endothermic, $\Delta H_{0}{ }^{0} \sim 0.51 \mathrm{eV}$, but has an activation energy of $\sim 1.8 \mathrm{eV}$ (for the ground-state species). ${ }^{31}$ Such a proposal appears attractive, both on energetic grounds and because of the obvious correlation between the relative strengths of the $\mathrm{CN}(B-X)$ and $\mathrm{C}_{2}(d-a)$ emission intensities. It also accords, qualitatively, with the observation (Fig. 4) that the fall in the density of emitting $\mathrm{C}_{2}(d)$ radicals (with increasing $x$ ) roughly complements the growth of $\mathrm{CN}(B)$ emission. Such an interpretation is unlikely to be the complete story, however-particularly when it comes to explaining production of the fastest emitting $\mathrm{CN}$ products.

Plume production and propagation through a background gas are often discussed in terms of a three-stage process: linear expansion, shock wave formation, and plume stopping. The first stage involves free expansion of the plume, with little influence from the background gas. Such is probably appropriate for the case of the species responsible for the $\mathrm{C}$ I and $\mathrm{C}$ II emissions above, the velocities of which show no obvious retardation with increasing $x$. New and/or enhanced emissions are observed away from the target surface, once some mixing of the plume species and the background gas has occurred-and assuming that the ensuing encounters result in reaction and/or excitation. This stage may involve formation of a shock front, if the background pressure is sufficiently high. Given the low $\mathrm{N}_{2}$ pressures in the 
present work, we neglect possible shock wave formation and use simple kinetic theory of gases arguments to estimate a few relevant time scales and distances. On this basis, the mean free path for an ablated particle propagating at $20 \mathrm{~km} \mathrm{~s}^{-1}$ in $20 \mathrm{mTorr}$ of $\mathrm{N}_{2}$ would be a few millimeters, and the mean time $(\tau)$ between collisions for such a particle will be 10-20 ns. For two background $\mathrm{N}_{2}$ gas molecules, the corresponding values are the same few millimeters and $\tau$ $\sim 5 \mu$ s. The $\mathrm{CN}^{*}$ emissions observed in the nanosecond PLA studies are seen to maximize at $x \sim 5 \mathrm{~mm}$ - as observed by others also. ${ }^{32-34}$ The apparent propagation velocities of the $\mathrm{CN}^{*}$ emitters are comparable to those deduced for the emitting $\mathrm{C}^{+}$ions and $\mathrm{C}$ neutrals, but about an order of magnitude faster than those of the observed $\mathrm{C}_{2}(d)$ radicals.

How should we interpret the apparent propagation velocity of $\mathrm{CN}^{*}$ radicals derived from plots like those in Fig. 9? As pointed out previously, ${ }^{35}$ the short radiative lifetime of the $\mathrm{CN}(B)$ state dictates that such excited radicals must emit almost as soon as they are formed, i.e., that the time-gated images of the $\mathrm{CN}(B-X)$ emission reveal the positions of successful $\mathrm{CN}(B)$ forming encounters during the time window of the observation. We note that the later-time $\lambda$ $>780 \mathrm{~nm}$ images in Fig. 5(c), which we attribute largely to $\mathrm{CN}(A-X)$ emission, appear somewhat fuzzier than the corresponding total OES images [Fig. 5(d), which are dominated by $\mathrm{CN}(B-X)$ emission]. Such is consistent with the longer $\tau_{\text {rad }}$ value of the $\mathrm{CN}(A)$ state, which allows some relaxation of the constraint that precludes the emitting product traveling any significant distance in the time between creation and emission.

Thus we view $\mathrm{CN}^{*}$ emission as a signifier of the first reactive encounter involving its $\mathrm{C}$-containing precursor generated in the PLA process. Contrary to at least one previous discussion, ${ }^{23}$ the "velocity" of the $\mathrm{CN}^{*}$ products deduced from the initial slope of a plot such as that shown in Fig. 9 does not provide a measure of the velocity (or thus of the kinetic energy) of the $\mathrm{CN}^{*}$ radicals themselves. Rather, they provide some measure of the velocity of the reactive precursor involved in $\mathrm{CN}^{*}$ radical formation. The "velocities" derived from analysis of the short-time images of both the $\mathrm{CN}(A)$ and $\mathrm{CN}(B)$ emissions exceed the velocity of the $\mathrm{C} \mathrm{I}$ emission observed in the nanosecond $248 \mathrm{~nm}$ PLA of graphite in vacuum. ${ }^{8}$ Conceivably, this could indicate that the faster fraction of the carriers responsible for the $\mathrm{C}$ I emission has a higher reaction probability with $\mathrm{N}_{2}$. We note, however, that the early time velocity of the $\mathrm{CN}(B)$ products matches well with that derived by monitoring the $\mathrm{C}$ II emission. Our earlier thesis that $\mathrm{C}$ II emission is a signifier of ablation products that begin life as $\mathrm{C}^{2+}$ ions and then undergo electron-ion recombination in the early stages of the expansion, thus leads to the suggestion that the faster $\mathrm{CN}^{*}$ products observed in the early time images are probably formed via abstractioncharge-transfer reactions such as ${ }^{36-38}$

$$
\mathrm{C}^{+}+\mathrm{N}_{2} \rightarrow \mathrm{N}^{+}+\mathrm{CN}
$$

or even, possibly, from $\mathrm{C}^{2+}+\mathrm{N}_{2}$ collisions. Reaction (2) is endothermic, $\Delta H_{0}{ }^{0} \sim 5.26 \mathrm{eV},{ }^{36}$ when all reactants and products are in their respective ground states. However, the $\mathrm{C}^{+}$ $\rightarrow \mathrm{C}^{2+}$ ionization energy is $24.38 \mathrm{eV} .^{39}$ The Rydberg states of
$\mathrm{C}^{+}$that are assumed to be present in the plume will thus have ample internal (electronic) energy to drive the forward reaction. As shown below, the likely collision energies are also more than sufficient to surmount an activation barrier of this magnitude. A recent study by Park and Lee ${ }^{40}$ lends support to this proposed mechanism. These workers investigated the OES accompanying nanosecond $266 \mathrm{~nm}$ PLA of graphite in a range of $\mathrm{N}_{2}$ pressures but, in contrast to the present study, mapped the emission in the $x y$ plane, by viewing down the $z$ axis. Spatially localized $\mathrm{C}_{2}(d-a)$ and $\mathrm{CN}(B-X)$ emissions were observed, at a number of different time delays. The former expanded more slowly, and died out more quickly — as in the present work. One other, significant, difference was apparent, however. The $\mathrm{C}_{2}(d-a)$ emission was observed to expand symmetrically about the surface normal $(x)$, but the $\mathrm{CN}(B-X)$ emission was found to be anisotropic about $x$ and to propagate along an axis inclined towards the axis of the incident laser beam. ${ }^{40}$ Such behavior has been seen previously, for the case of the $\mathrm{C}$ II emissions arising in the nanosecond $193 \mathrm{~nm}$ PLA of graphite in vacuum, and rationalized in terms of preferential multiphoton excitation and ionization of $\mathrm{C}$ species in the leading part of the expanding plasma ball that are exposed to the greatest incident photon flux, and subsequent EIR and radiative decay. ${ }^{26}$ Such anisotropy could not be seen in the present study because of the viewing geometry, whereby the incident laser axis projects onto the surface normal. Any asymmetry evident in the present images [see, e.g., Fig. 5(a)] is attributed to the inhomogeneous spatial profile of the nanosecond excimer laser output.

Given the foregoing interpretation of the apparent velocities of the $\mathrm{CN}^{*}$ emitters, there remains a question as to what might be the real impact velocity of such species on the surface of the substrate on which a $\mathrm{CN}_{x}$ film is grown. Analyses of images of $\mathrm{CN}^{*}$ emission taken at later $t$ (the $x_{\text {peak }}$ data from which are summarized in Fig. 9) imply much slower expansion velocities, but still do not provide any clear answer. The substrate in our experiments is positioned at $x$ $=30 \mathrm{~mm}$, and inspection of $I$ vs $x$ plots such as those shown in Fig. 7(c) shows a definite enhancement in the $\mathrm{CN}^{*}$ emission intensity at larger $x$ in images recorded at later $t$. As noted previously, ${ }^{23}$ the presence of the substrate introduces several possible secondary excitation mechanisms. For example, collision of a gas phase species with the substrate surface offers a route for converting the incident kinetic energy of the former into internal (electronic, vibrational, rotational) excitation. Such gas-surface collisions can also lead to compaction of previously deposited material within the film, and to the sputtering of such material back into the gas phase. Purely gas phase collisions involving an incident species and one that has scattered from the surface will involve higher than average collision energies. All such processes could lead to an increase in optical emission in the vicinity of the substrate; the relative intensities, and the spatial extent, of any such emissions will vary with $t$ because of the time dependence of the density, composition, and energy content of the incident pulse of gas created by the PLA event (and modified by subsequent gas phase collisions).

One estimate of the average velocity with which $\mathrm{CN}$ 
radicals strike the substrate can be obtained from the collision frequency and mean-free-path estimates quoted previously. Let us start by assuming that fast $\mathrm{CN}^{*}$ radical formation is via the $\mathrm{C}^{+}+\mathrm{N}_{2}$ abstraction-charge-transfer reaction (2), that the initial $\mathrm{C}^{+}$ion velocity $v_{x}=20 \mathrm{~km} \mathrm{~s}^{-1}$ along the target surface normal and, for simplicity, that the background $\mathrm{N}_{2}$ molecules are stationary. In this model, the laboratory and center-of-mass frames are equivalent, and the initial $\mathrm{C}^{+}+\mathrm{N}_{2}$ collision energy $\left(E_{\text {coll }} \sim 17.5 \mathrm{eV}\right)$ is more than sufficient to overcome the endothermicity of reaction (2). The $\mathrm{C}^{+}$ions seen in the OES carry electronic energy also, but we assume that most of the $\mathrm{C}^{+}$ions available to participate in reaction (2) are in their ground state, so we ignore possible additional (electronic) contributions to the energy available for reaction. Given these assumptions, the energy available to the $\mathrm{N}^{+}$ $+\mathrm{CN}$ products is $\sim 12.1 \mathrm{eV}(\sim 8.9 \mathrm{eV}$ in the case that the $\mathrm{CN}$ products are in the $v=0$ level of the $B$ state). If all of this available energy is partitioned into product translation, the resulting $\mathrm{CN}(B)$ fragments can have any velocity in the range of $10.8 \geqslant v_{x} \geqslant 1.2 \mathrm{~km} \mathrm{~s}^{-1}$, where the limits correspond to, respectively, pure stripping and pure rebound dynamics. These numbers are meant to be illustrative only. Clearly, some reactive encounters will involve greater initial energies (either from reactant electronic excitation, or as a result of a higher impact kinetic energy) but equally, in many cases, more of the reaction exoergicity than has been assumed here will be channeled into non-translational degrees of freedom [e.g., $\mathrm{CN}(B)$ vibrational excitation or electronic excitation of the $\mathrm{N}^{+}$cofragment]. Given a target-substrate separation of $30 \mathrm{~mm}$ and an estimated mean free path in $20 \mathrm{mTorr}$ of $\mathrm{N}_{2}$ of $5 \mathrm{~mm}$, the resulting $\mathrm{CN}$ radical is likely to suffer several further (non-reactive) collisions-most probably with background $\mathrm{N}_{2}$ molecules-before striking the substrate. If we persist with the foregoing simplifications, momentum conservation could cause $\left\langle v_{x}\right\rangle$ to be reduced by as much as $50 \%$ in each subsequent collision. Thus it is difficult to envisage that the average impact velocity of $\mathrm{CN}$ radicals striking a substrate $30 \mathrm{~mm}$ distant from a graphite target subjected to nanosecond $248 \mathrm{~nm}$ PLA in 20 mTorr of $\mathrm{N}_{2}$ will be more than a few $\mathrm{km} \mathrm{s}^{-1}\left(v_{x}=5 \mathrm{~km} \mathrm{~s}^{-1}\right.$ equates to an impact energy of $\sim 7 \mathrm{eV}$ ).

In closing this discussion of $\mathrm{CN}$ radical formation, propagation, and impact on the substrate surface we recall that $\mathrm{C}$ I emissions also contribute to the observed OES. The ultimate result of such emissions will be formation of ground-state $\mathrm{C}$ atoms. Reaction (3), the activation energy for which has been estimated at $\sim 1.9 \mathrm{eV},{ }^{31,41}$ thus represents another potential source of $\mathrm{CN}$ radicals,

$$
\mathrm{C}+\mathrm{N}_{2} \rightarrow \mathrm{CN}+\mathrm{N} \text {. }
$$

The total $\mathrm{C}$ atom density in the ablation plume is likely to be far higher than the $\mathrm{C}^{+}$ion density. Recall that the EIR interpretation associates $\mathrm{C}$ I emissions with species created as $\mathrm{C}^{+}$ ions in the initial PLA event. Charged species experience an initial (Coulombic) acceleration not available to neutral ejecta, and will thus typically exhibit higher recoil velocities. The $\mathrm{C}$ atoms available to react with background $\mathrm{N}_{2}$ gas molecules are thus expected to have a broad velocity distribution, with a faster fraction deriving from $\mathrm{C}^{+}$ions that have undergone EIR and subsequent relaxation, and a slower component that was created as neutral atoms. $E_{\text {coll }}$ for the faster fraction of $\mathrm{C}+\mathrm{N}_{2}$ collisions may well be $>10 \mathrm{eV}$, so it would be foolish to exclude reaction (3) as another probable source of $\mathrm{CN}$ radicals available for incorporation in any eventual $\mathrm{CN}_{x}$ film. That said, it is hard to see how $\mathrm{CN}$ radicals formed via this route could have incident kinetic energies larger than those deduced in the foregoing consideration of the $\mathrm{C}^{+}+\mathrm{N}_{2}$ reaction products. Reaction (1) is unarguably capable of producing $\mathrm{CN}$ radicals also, but the slow recoil velocities deduced for the emitting $\mathrm{C}_{2}$ species in the plume suggest that these will, if anything, have even smaller $v_{x}$ values.

$\mathrm{N}_{2}{ }^{+}(B-X)$ emission is evident in the OES accompanying nanosecond $248 \mathrm{~nm}$ PLA of graphite in $\mathrm{N}_{2}$, and dominates the emission seen in the femtosecond studies. Femtosecond PLA releases much less carbon per pulse, so the relative yield of $\mathrm{C}_{2}$ radicals (which are considered to arise via recombination of gas phase $\mathrm{C}$ atoms) would be expected, and is observed, to be much lower than in the corresponding nanosecond study. Femtosecond PLA involves much higher peak intensities, and the resulting plume is generally more highly ionized. Thus the plume formed via femtosecond PLA should contain a higher $\mathrm{C}^{n+} / \mathrm{C}$ ratio $(n \geqslant 1)$-a prediction in accord with the present OES data, and with femtosecond PLA studies of graphite in Ar (Ref. 19) and in vacuum. ${ }^{8}$ $\mathrm{C}^{n+}+\mathrm{N}_{2}$ collisions should thus be relatively more important in the case of femtosecond PLA. We have argued that $\mathrm{C}^{+}$ $+\mathrm{N}_{2}, \mathrm{C}+\mathrm{N}_{2}$, and $\mathrm{C}_{2}+\mathrm{N}_{2}$ reactive encounters probably all contribute to the $\mathrm{CN}^{*}$ emission seen in the nanosecond PLA studies. The $\mathrm{CN}^{*}$ emission following femtosecond PLA is much weaker-reflecting the reduced contributions from, especially, reactions (1) and (3). Previous guided ion mass spectrometry studies of the $\mathrm{C}^{+}+\mathrm{N}_{2}$ reaction have revealed a substantial role for the charge-transfer channel

$$
\mathrm{C}^{+}+\mathrm{N}_{2} \rightarrow \mathrm{C}+\mathrm{N}_{2}^{+},
$$

at all collision energies above threshold $\left[\Delta H_{0}{ }^{0}=4.32 \mathrm{eV}\right.$ for all ground-state species, and $7.48 \mathrm{eV}$ for ground-state reactant forming $\mathrm{N}_{2}^{+}(B)$ ]; this channel becomes totally dominant at $E_{\text {coll }} \sim 20 \mathrm{eV}$. ${ }^{36}$ We have found no detailed studies of the way in which $\mathrm{C}^{+}$electronic excitation affects the product branching, or of the related reaction involving $\mathrm{C}^{2+}$ ions. It is thus tempting to attribute the dominance of $\mathrm{N}_{2}{ }^{+}(B-X)$ emission in the OES following femtosecond PLA of graphite in $\mathrm{N}_{2}$ to reaction (4). The comparatively slow speed with which this localized emission expands, and its persistence, both merit comment, however. Inspection of Fig. 9(b) suggests some curvature of the $x_{\text {peak }}$ vs $t$ plot for the $\mathrm{N}_{2}{ }^{+}(B-X)$ emission; the gradient first increases and then appears to decrease with increasing $t$. The deduced propagation speed at early times is, in fact, rather similar to that predicted by the previous momentum conservation arguments, and could be reasonable if charge transfer is favored by a stripping-type mechanism, and less momentum is thus transferred to the resulting $\mathrm{N}_{2}^{+}$ion. If this ion was formed in its $B$ state, it should decay radiatively, within $\sim 60$ ns -i.e., essentially at the point where the charge transfer occurred. This mechanism alone is thus unable to account for the persistence of 
the $\mathrm{N}_{2}{ }^{+}(B-X)$ emission, which is observable at $>1 \mu \mathrm{s}$ after the PLA event.

We recognize that $\mathrm{C}^{2+}+\mathrm{N}_{2}$ charge-transfer collisions could offer a route to $\mathrm{N}_{2}{ }^{+}(B)$ products. We also recognize that the present observations could be explained by invoking population of a higher-energy, metastable, state of $\mathrm{N}_{2}^{+}$, which feeds into the (observed) $\mathrm{N}_{2}{ }^{+}(B)$ state over an extended time scale. In the absence of additional information about $\mathrm{C}^{2+}+\mathrm{N}_{2}$ reaction mechanisms, and/or about suitable metastable excited states of $\mathrm{N}_{2}{ }^{+}$, however, we consider the following to be the most plausible explanation for the present observations: Femtosecond PLA leads to a relatively low (in comparison with nanosecond PLA) yield of ejected material per pulse, comprising neutrals, $\mathrm{C}^{n+}$ ions, and electrons. Charge transfer results in $\mathrm{N}_{2}{ }^{+}$ion formation, and rapid depletion of the $\mathrm{C}^{n+}$ number density-at small $x$. Some momentum is transferred in the charge-transfer process, so the resulting $\mathrm{N}_{2}{ }^{+}$ions acquire an expansion velocity. The electrons formed in the ablation process track the positive charges, which now reside with the expanding cloud of $\mathrm{N}_{2}{ }^{+}$ions (ambipolar expansion). Numerous studies, involving a wide range of laboratory and astrophysical plasmas, have demonstrated that electron collisions with $\mathrm{N}_{2}{ }^{+}$ions lead to intense $\mathrm{N}_{2}^{+}(B-X)$ emission. Calculated electron-impact excitation cross sections $^{42}$ for this process accord with the observed dominance of the $v^{\prime}=0 \rightarrow v^{\prime \prime}=0$ band at $391.4 \mathrm{~nm}$. The charge-transfer mechanism (4), followed by one (or possibly more) subsequent electron-impact excitations, can also account for the modest $\mathrm{N}_{2}{ }^{+}$rotational excitation deduced from the simulations of the observed OES. Electron collisions with neutral $\mathrm{N}_{2}$ molecules can also lead to $\mathrm{N}_{2}^{+}(B-X)$ emission if the collision energies are sufficiently high; such excitations may gain in importance at larger $x$ as the density of $\mathrm{N}_{2}{ }^{+}$falls (as it must do if any of the electron-ion encounters result in a dissociative recombination rather than excitation) and might help to explain both the persistence of this particular emission and the observed slowing of the $\mathrm{N}_{2}^{+}(B-X)$ emission at large $x$.

\section{CONCLUSION}

Fundamental aspects of the nanosecond and femtosecond $248 \mathrm{~nm}$ PLAs of graphite in low pressures of $\mathrm{N}_{2}$ have been investigated by wavelength-, space-, and time-resolved optical emission spectroscopy and by analysis of the resulting $\mathrm{CN}_{x}$ films. Nanosecond laser excitation provided $\sim 5 \times$ higher pulse energies, but the superior beam quality of the femtosecond pulse enabled comparative studies at similar incident fluences (though, necessarily, very different incident intensities). Nanosecond PLA results in significantly more material removal per pulse. Laser Raman spectroscopy confirms successful incorporation of nitrogen within the films grown by both nanosecond and femtosecond PLAs; the former films are deduced to be both thicker and to have higher $\mathrm{N}$ content. OES studies of the nanosecond ablation plume reveal C I, C II, and $\mathrm{C}_{2}$ emissions-concentrated close to the target—and, at larger $x$, weak $\mathrm{N}_{2}{ }^{+}$and strong $\mathrm{CN}$ emissions. In contrast, wavelength-resolved spectra of the plume accompanying femtosecond PLA are dominated by
$\mathrm{N}_{2}{ }^{+}(B-X)$ emission. Analysis of time-gated images of the respective emissions has allowed estimation of the propagation speeds of the various carriers, and revealed contributions from secondary excitation and/or material sputtering near the surface of the depositing film. Possible production routes for secondary emitters such as $\mathrm{CN}$ and $\mathrm{N}_{2}^{+}$are discussed. Observation of $\mathrm{CN}(B-X)$ emission confirms $\mathrm{C} \equiv \mathrm{N}$ bond formation in the gas phase. Arguments are presented to demonstrate that any apparent propagation "velocity" deduced by analysis of such secondary emission is unlikely to provide a useful measure of the kinetic energy with which such carriers impact on the growing film.

\section{ACKNOWLEDGMENTS}

The authors are very grateful to Dr. D. Anglos and Professor C. Fotakis for their support of this work, the major part of which was performed at the Ultraviolet Laser Facility operating at IESL FORTH, Heraklion, and supported by the EU through the Research Infrastructures activity of FP6 (Contract No. RII3-CT-2003-506350: Laserlab-Europe). The authors are also grateful to Professor A. J. Orr-Ewing, Dr. J. A. Smith, Dr. C. M. Western (Bristol), A. Egglezis, and A. Klini (FORTH) for their many contributions to the work described herein, and to EPSRC for funding via two portfolio grants (LASER and Integrated Electronics) and the Carbon Based Electronics Initiative.

${ }^{1}$ C. W. Ong, X.-A. Zhao, Y. C. Tsang, C. L. Choy, and P. W. Chan, Thin Solid Films 280, 1 (1996).

${ }^{2}$ J. P. Zhao, Z. Y. Chen, T. Yano, T. Ooie, and M. Yoneda, J. Appl. Phys. 89, 1580 (2001).

${ }^{3}$ E. Gyorgy, V. Nelea, I. N. Mihailescu, A. Perrone, H. Pelletier, A. Cornet, S. Ganatsios, and J. Werckmann, Thin Solid Films 388, 93 (2001).

${ }^{4}$ J. Neidhardt, L. Hultman, E. Broitman, T. W. Scharf, and I. L. Singer, Diamond Relat. Mater. 13, 1882 (2004).

${ }^{5}$ N. Hellgren, M. P. Johansson, E. Broitman, P. Sandstrom, L. Hultman, and J.-E. Sundgren, Thin Solid Films 382, 146 (2001).

${ }^{6}$ S. Muhl and J. M. Mendez, Diamond Relat. Mater. 8, 1809 (1999).

${ }^{7}$ S. E. Rodil and S. Muhl, Diamond Relat. Mater. 13, 1521 (2004).

${ }^{8}$ F. Claeyssens, M. N. R. Ashfold, E. Sofoulakis, C. G. Ristoscu, D. Anglos, and C. Fotakis, J. Appl. Phys. 91, 6162 (2002).

${ }^{9}$ P. S. Banks, L. Dinh, B. C. Stuart, M. D. Feit, A. M. Komashko, A. M. Rubenchik, M. D. Perry, and W. McLean, Appl. Phys. A: Mater. Sci. Process. 69, S347 (1999).

${ }^{10}$ A. S. Loir et al., Appl. Surf. Sci. 208, 553 (2003).

${ }^{11}$ M. D. Shirk and P. A. Molian, Carbon 39, 1183 (2001).

${ }^{12}$ M. Okoshi, S. Higuchi, and M. Hanabusa, J. Appl. Phys. 86, 1768 (1999).

${ }^{13}$ S. Rey, F. Antoni, B. Prevot, E. Fogarassy, J. C. Arnault, J. Hommet, F. Le Normand, and P. Boher, Appl. Phys. A: Mater. Sci. Process. 71, 433 (2000).

${ }^{14}$ Z. Geretovszky, Z. Kantor, and T. Szorenyi, Appl. Surf. Sci. 208, 547 (2003).

${ }^{15}$ T. Szorenyi, E. Fogarassy, C. Fuchs, J. Hommet, and F. Le Normand, Appl. Phys. A: Mater. Sci. Process. 69, S941 (1999).

${ }^{16}$ Z. Geretovszky, Z. Kantor, I. Bertoti, and T. Szorenyi, Appl. Phys. A: Mater. Sci. Process. 70, 9 (2000).

${ }^{17}$ S. Acquaviva, A. Perrone, A. Zocco, A. Klini, and C. Fotakis, Thin Solid Films 373, 266 (2000).

${ }^{18}$ S. J. Henley, G. M. Fuge, and M. N. R. Ashfold, J. Appl. Phys. 97, 023304 (2005).

${ }^{19}$ S. J. Henley, J. D. Carey, S. R. P. Silva, G. M. Fuge, M. N. R. Ashfold, and D. Anglos, Phys. Rev. B 72, 205413 (2005).

${ }^{20}$ K. P. Huber and G. Herzberg, Molecular Spectra and Molecular Structure IV. Constants of Diatomic Molecules (van Nostrand, New York, 1979).

${ }^{21}$ F. J. LeBlanc, J. Chem. Phys. 48, 1980 (1968).

${ }^{22} \mathrm{C}$. M. Western, PGOPHER, a program for simulating rotational structure, University of Bristol, http://pgopher.chem.bris.ac.uk 
${ }^{23}$ A. A. Voevodin, J. G. Jones, J. S. Zabinski, and L. Huttman, J. Appl. Phys. 92, 724 (2002).

${ }^{24}$ G. M. Fuge, C. J. Rennick, S. R. J. Pearce, P. W. May, and M. N. R. Ashfold, Diamond Relat. Mater. 12, 1049 (2003), and references therein.

${ }^{25}$ A. C. Ferrari and J. Robertson, Phys. Rev. B 61, 14095 (2000), and references therein.

${ }^{26}$ F. Claeyssens, R. J. Lade, K. N. Rosser, and M. N. R. Ashfold, J. Appl. Phys. 89, 697 (2001).

${ }^{27}$ R. K. Thareja, R. K. Dwivedi, and K. Ebihara, Nucl. Instrum. Methods Phys. Res. B 192, 301 (2002)

${ }^{28}$ S. Abdelli-Messaci, T. Kerdja, A. Bendib, and S. Malek, J. Phys. D 35, 2772 (2002).

${ }^{29}$ S. Wee and S. M. Park, Opt. Commun. 165, 199 (1999).

${ }^{30}$ C. Vivien, J. Hermann, A. Perrone, C. Boulmer-Leborgne, and A. Luches, J. Phys. D 31, 1263 (1998).

${ }^{31}$ T. Sommer, T. Kruse, P. Roth, and H. Hippler, J. Phys. Chem. A 101, 3720 (1997).

${ }^{32}$ S. Trusso, E. Barletta, F. Barreca, and F. Neri, Appl. Phys. A: Mater. Sci.
Process. 79, 1997 (2004).

${ }^{33}$ S. Acquaviva and M. L. De Giorgi, J. Phys. B 35, 795 (2002).

${ }^{34}$ E. Aldea, A. P. Caricato, G. Dinescu, A. Luches, and A. Perrone, Jpn. J. Appl. Phys., Part 1 36, 4686 (1997).

${ }^{35}$ K. L. Saenger, J. Appl. Phys. 66, 4435 (1989).

${ }^{36}$ J. D. Burley, L. S. Sunderlin, and P. B. Armentrout, J. Chem. Phys. 94, 1939 (1991).

${ }^{37}$ R. C. C. Lao, R. W. Rozett, and W. S. Koski, J. Chem. Phys. 49, 4202 (1968).

${ }^{38}$ Ch. Ottinger and J. Simonis, Chem. Phys. 28, 97 (1978).

${ }^{39}$ NIST Atomic Spectra Database, http://physics.nist.gov/cgi-bin/AtData/ main_asd

${ }^{40}$ S. M. Park and K. H. Lee, Appl. Surf. Sci. 178, 37 (2001).

${ }^{41}$ A. J. Dean, R. K. Hanson, and C. T. Bowman, J. Phys. Chem. 95, 3180 (1991).

${ }^{42}$ O. Nagy, C. P. Balance, K. A. Berrington, P. G. Burke, and B. M. McLaughlin, J. Phys. B 32, L469 (1999). 